\title{
Ensemble EMD based Time-Frequency Analysis of Continuous Adventitious Signal Processing
}

\author{
B.B Shankar ${ }^{1^{*}}$, D. Jayadevappa ${ }^{2}$ \\ ${ }^{1}$ Jain University, Bengaluru, Karnataka, India \\ ${ }^{2}$ Department of EIE, JSS ATE, Bangalore, Karnataka India \\ *Corresponding author E-mail: sankrubb@gmail.com
}

\begin{abstract}
The importance of lung sound analyses is increasing day by day very rapidly. In this paper, we present a new method for analysis of two classes of lung signals namely wheezes and crackles. The procedure used in this article is based on improved Empirical Mode Decomposition (EMD) called Ensemble Empirical Mode Decomposition (EEMD) to analyze and compare continuous and discontinuous adventitious sounds with EMD. These two proposed procedures decompose the lung signals into a set of instantaneous frequency components. Function (IMF). The continuous and discontinuous adventitious sounds are present in an asthmatic patient, produces a non-stationary and nonlinear signal pattern. The empirical mode decomposition (EMD) decomposes such characteristic signals. The instantaneous frequency and spectral analysis related to dual techniques specified above are utilized by IMF to investigate and present the outcome in the timefrequency distribution to investigate the qualities of inbuilt properties of lung sound waves. The Hilbert marginal spectrum has been used to represent total amplitude and energy contribution from every frequency value. Finally, the resultant EEMD analysis is better for wheezes that solves mode mixing issues and improvisation is seen over the EMD method.
\end{abstract}

Keywords: Adventitious, EMD, Hilbert spectral analysis, Wheezing, Crackle.

\section{Introduction}

The time-frequency analysis of adventitious sounds became more popular to achieve high accuracy of the diagnosed result. From Taplidou's era [12] time -frequency-based analysis of lung sounds has been designed. Thus, presented procedure utilizes a Short Time Fourier Transform which leaves the drawback of pathological and high noise robustness. The detection of robustness of different adventitious sounds well analyzed and presented [13]. Unfortunately, this could diagnose only internal noises leaving external noises unaltered. The respiratory sound wave (RSW) is multicomponent, nonlinear, and non-stationary signals. This RSW consists of normal RSWS and abnormal RSW that is superimposed to RSW. There are mainly two types of abnormal RSWS are discussed in this context. The continuous adventitious sound RSW (CASRSW) as their duration is more than 85 milliseconds. The human ear can hear easily as the frequency range is between 100 $\mathrm{Hz}$ and $1 \mathrm{kHz}$. Thus, we can see the sharp peaks in the power spectrum. If the pitch of the wheeze is too low, then it usually referred as rhonchi (R A L E. "Adventitious Sounds") and discontinuous adventitious sound RSW (DASRSW). Normal RSW is random in nature, whereas CASRSW is quasi-periodic waveforms with a duration of more than $80-100 \mathrm{~ms}$ and a fundamental frequency of over $100 \mathrm{~Hz}$, and DASRSW are transient and short sounds (around $20 \mathrm{~ms}$ ), with high-frequency components (above $300 \mathrm{~Hz}$ ) [4,5]. Adventitious sounds are usually detected more rapidly indicating abnormality of the respiratory system. The respiratory waveform of abnormality gives many variations in the wave characteristics of bronchial and vesicular sounds but only a few are widely recognized as there is a difficulty in verbal description. Coarse lung sounds, for example, indicates lower frequencies are accumulated and some of the higher frequencies are lost. The terms like cavernous, amphoric, conical and cogwheel also used but nowadays these are ignored as they are not having any clinical importance. As asthma becomes severe the wheezing is observed at the site chest. The variations in the normal respiratory sound exhibiting high pitched musical sounds which are produced in the airway is called adventitious sound. Adventitious sound can be noticed by a human acoustic system usually with inspiration and expiration stages. These adventitious sounds are continuous and discontinuous. The continuously generating adventitious sounds are called continuous adventitious sound (CAS), wheezes are the examples of CAS. The adventitious sounds that occur only for a certain period of time dis-continuously are called discontinuous adventitious sound (DAS). The crackles are the examples of DAS and crackles are of two types, fine and coarse crackles. The discontinuous adventitious sounds (DAS) are short, popping sound signals. The CAS is low or piercing sound wave created at the time of termination phase of breathing. The 'Rhonchi in the bronchi', the rhonchi is heard in bronchi. The Hilbert - Huang Transformation is the exceptionally successful nonlinear frequency verification tool for adventitious sound waves. The Fourier analysis technique is appropriate if the wave is linear and stationary. The inbuilt segments of unusual irregular sound waves must be separated and broke down precisely using time-frequency mode of examination. The non-stationary sound wave examination using wavelet transform and spectral analysis method is a suitable approach, But HHT calculation is utilized for agreeable precise outcomes. The energy level spectrogram is also plotted with the help of Hilbert spectral analysis for better examination. The review of adventitious sound regarding spectrum analysis in control after some time is accomplished by wavelet transform which gives an ideal harmony among temporal and frequency exactness. The 
wavelet transform is certifiably not a reasonable system for nonlinear and nonstationary oscillatory-like waveforms from the adventitious information, in such circumstance empirical mode decomposition (EMD) of Hilbert-Huang Transform (HHT) which is iterative method finds the most encouraging procedure. It gives the particular level of frequencies extend that can't be anticipated ahead of time by us. These frequencies information have a considerable measure of intensity and are about segments of the previous frequency components were separated. The specific procedure which deals with fine data implanted inside an adventitious sound must be broken down into Intrinsic Mode Function (IMF) which is a fundamental building block of EMD. The EMD and Ensemble EMD (EEMD) based Hilbert transform is a useful procedure to set up CAS more precisely than another customary time-frequency investigation [3]. The EMD and EEMD occupying top priority techniques among time-frequency investigation procedure contrast with STFT, wavelets and any type of Fourier Transformation methods. The decomposition of sinusoids of frequency domain gives unacceptable data and side lobes ancient rarities. In this paper, the analysis technique finds the envelopes among minima and maxima alongside residuals and IMF's segments. It finds a reasonable position among different adventitious sound investigation system and in the meantime as it is a specific procedure expends additional time length to process the whole 20 -second span wave. However, the sound rush of a specific pitch length is considered to keep away from delay. The upgraded EMD and EEMD with noise-free level are accomplished.

\section{Materials and Methods}

The different wheezing and crackles are collected from online at http://www.easyauscultation.com/unusual lung-sounds and few of them have been used for the investigation. The concise portrayal about the distinctive crackles and wheezes likewise gave in this dataset. The adventitious waves are for various phases of CAS and DAS sound waves and are typically semi-occasional. The waves so gathered are having complete wave span of 20 seconds and of 160000 samples with the sampling rate of $8000 \mathrm{~Hz}$. The amplitude variation lies in the middle of $-100 \mathrm{~dB}$ and $+120 \mathrm{~dB}$ and it is noticed by dual algorithms. These are adequate for the proposed procedure and are developed utilizing MATLAB for analysis.

\subsection{Empirical mode decomposition}

The various maxima and minima are dependent on individual IMF and its sampling frequency. The mean values envelope is estimated by zero level of maxima and minima. The basic step in case of EMD to find out local minima and local maxima of an adventitious waveform. The smooth envelope is formed by connecting all maxima and minima components, i.e. $\operatorname{Emax}(t)$ and $\operatorname{Emin}(t)$ respectively [9].

Mean value, $m(t)$ is given by the following equation.

$$
m(t)=\bmod \left[E_{\max (t)}+E_{\min (t)}\right] / 2
$$

The above equation may be subtracted by original adventitious wave $\mathrm{x}(\mathrm{t})$ to get desired IMF component and procedure must be repeated for all the IMFs. The residual component is also analyzed after analyzing all IMFs obtained by the procedure.

\subsection{Ensemble EEMD}

To eliminate certain aliasing effect and also to provide better physical meaning for individual IMF, the Empirical Mode Decomposition (EMD) has been proposed recently (Huang, et al. 1998, Huang et al. 1999) as an adaptive time-frequency data analysis method [10]. This is the ensemble copy of EMD gives the 'true' modes. The EEMD algorithm initially generates zero means with white noise realization. Next, decomposes completely into $\mathrm{k}$ modes, i.e. $\mathrm{dk}$, where $\mathrm{k}$ gives mode. The averaging of the corresponding modes can be obtained after sifting iterations of various numbers [8]. EEMD fairly solves the mode mixing phenomenon in adventitious sound waves. EEMD accurately decomposes such sound waves into simple IMFs. EEMD accurately analyses and restores the time-frequency distribution of the adventitious sound waves. To overcome the scale separation problem a new ensemble technique which is upgraded method of EMD is proposed, the Ensemble EMD (EEMD), which defines the true IMF components as the mean of an ensemble of trials, each consisting of the signal plus a white noise of finite amplitude. The CEEMDGN utilizes the last mode of each iteration. The local mean of Gaussian noise thus defines true mode [8]. The instantaneous frequency can be found if time derivative of the phase of complex wave sound is considered. The experimental way of getting such a complex wave from the real one is considering the analytic wave. Analytic signal, Ai(t), from individual IMF, $\operatorname{Ii}(t)$ is.

$A(i)=I_{i}(t)+j H\left[I_{i}(t)\right]=b_{i}(t) e^{(j ø i(t))}$

Where H[.] indicates Hilbert Transform, $b i(t)$ is the constant absolute parameter of $A i(t)$ and $\Phi i(t)$. After obtaining the analytic wave from individual IMF, $I F(t)$ can be found by the phase derivative of the analytic sound wave.

Thus, EEMD performs ensemble empirical decomposition, especially a Gaussian noise-based algorithm. The EEMD gives the suitable results when white noise and certain amplitudes are added by decomposing through EMD. If this is repeated several times, then the average IMF straight away exhibits the "true" IMF set [10]. The discrete wavelet transform decomposes adventitious sound waves into basic building blocks called wavelets. The excellent Hilbert spectrum gives the time localization of the wave frequency [15].

$$
I F(t)=\frac{1}{2 \pi} \frac{d \emptyset i(t)}{d t}
$$

\section{Results}

The plots of the adventitious sound wave with its 14 IMFs along with the residuals shown in the following figures.

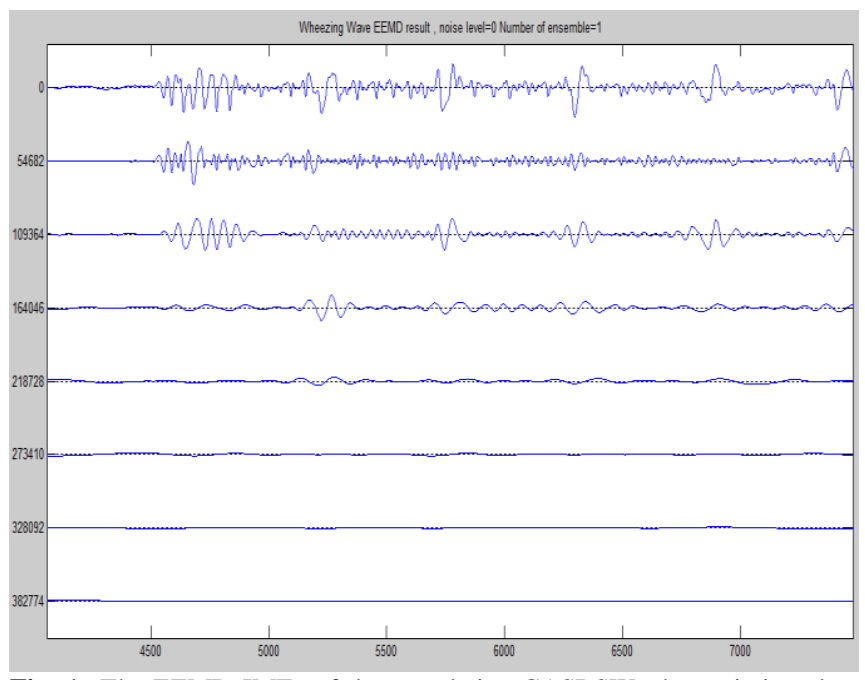

Fig. 1: The EEMD IMFs of the correlative CASRSW: the variations by the wheezing wave can be seen in the almost regular amplitude variations in the first three IMFs (from the top down) 


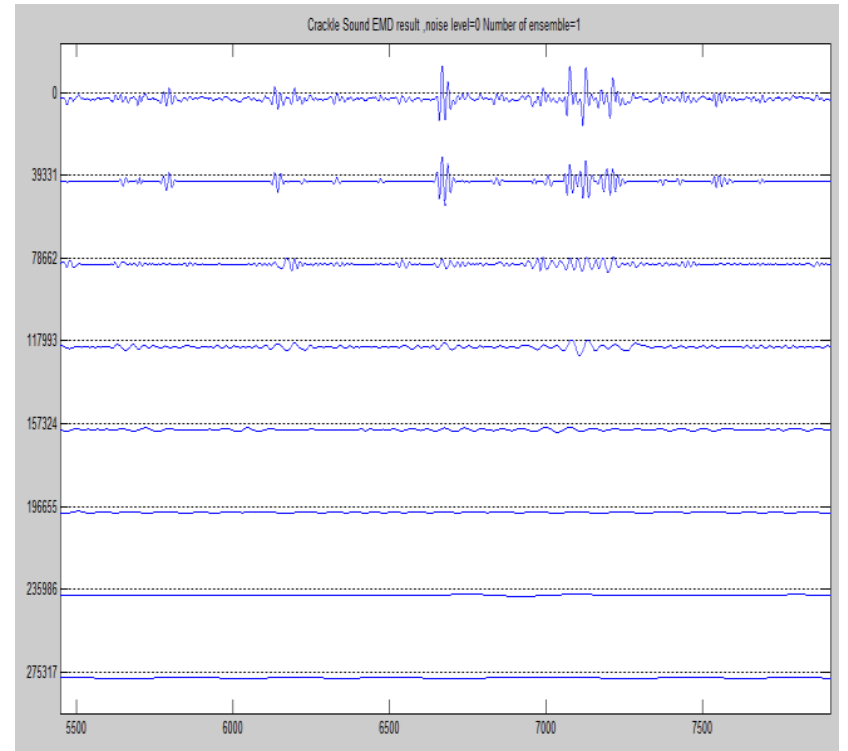

Fig. 2: The EEMD IMFs of the correlative DASRSW: the variations by the wheezing wave can be seen in the almost regular amplitude variations in the first three IMFs (from the top down)

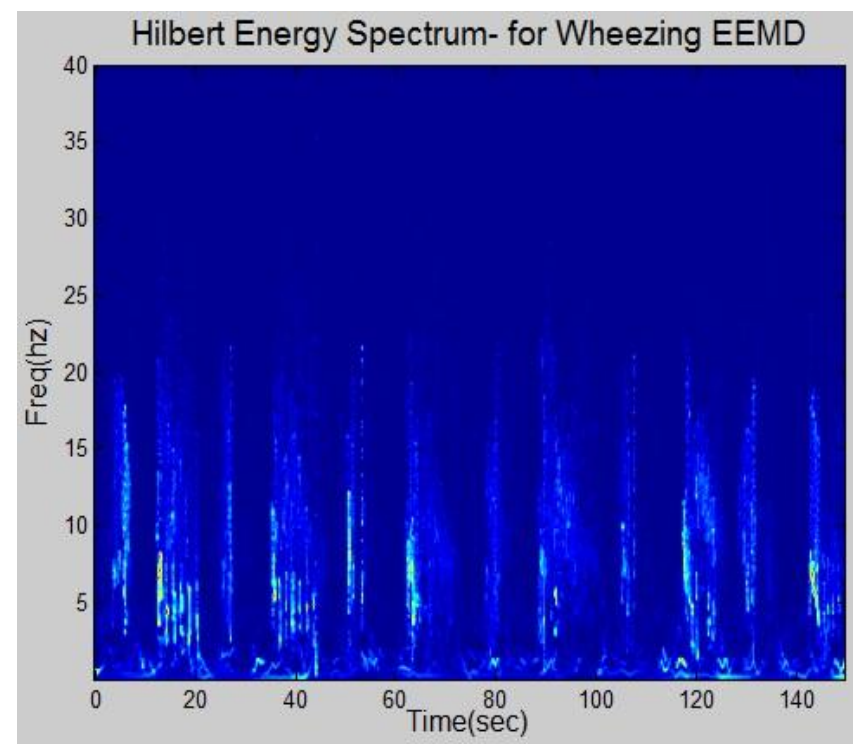

Fig. 3: The Hilbert energy spectrum with EEMD for CASRSW

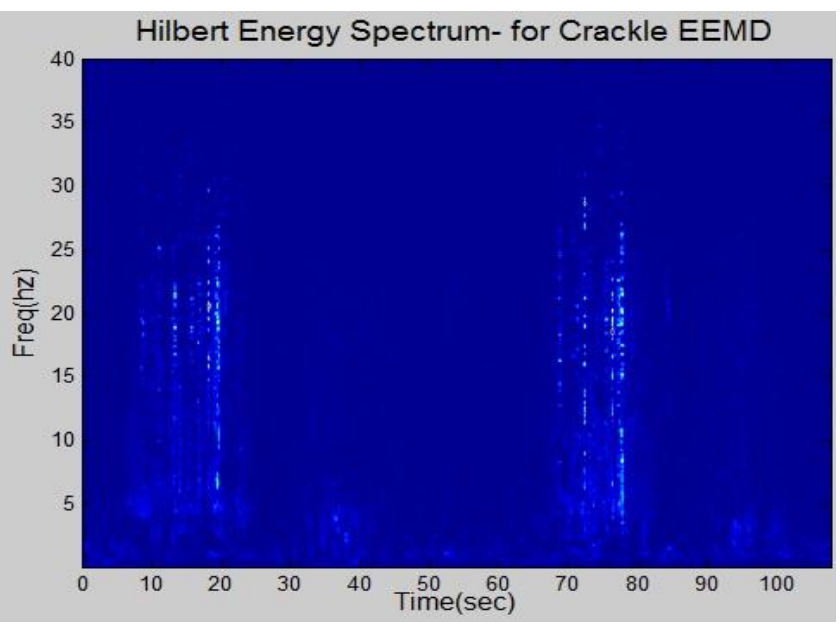

Fig. 4: The Hilbert energy spectrum with EEMD for DASRSW

The first wave plotted in figures 1 and 2 shows the original wheezing and crackle waves and residual at the end of these figures respectively. Totally there are 14 increasing IMFs are plotted with zero noise level and the total number of ensembles detected is one.
These are digitized at $10,000 \mathrm{~Hz}$. The IMFs of the adventitious sound activity is clearly visible in the first six components in case of wheezing and in the first four components crackles. The decomposition of CASRSW with its mean and sampling frequency for each IMF is determined and initial IMF for the first sampling frequency is calculated and its value is $54682 \mathrm{~Hz}$. Each successive IMF contains oscillating lower frequency waves. However, CASRSW shows varying amplitudes and frequency on the time axis. The comparatively high pitched but at different instant of time is observed for DASRSW. The statistical features of these instants are calculated. The first wave in fig. 1 shows the original wheezing wave and last wave in fig. 1 indicates residual. The different IMFs are calculated and plotted to start from the sampling frequency of 39331 to 550634. The minima and maxima for ASRSW are estimated and calculated for succeeding IMFs. The results clearly exhibit the EEMD has the special characteristic feature of exploring the information that manifests the hidden physical phenomenon. The Gaussian noise which acts as reference scale ensemble with EMD to overcome unstable noise sensitivity and mode mixing effect. The figures show even the ensemble mean of each IMF. The Hilbert spectra give the detailed distribution of energy. The improved quality of the IMF has greater effects on the time-frequency distribution of the wave in Hilbert spectra format is shown figures 3 and 4 . The energy spectra are plotted separately, and one can notice the continuous spectra in case of CASRSW and this is continuous wheezing signal encountered in an asthmatic patient. These figures give the timefrequency distribution of such wheezes in terms of spectrum.

The mean and standard deviation at the different instance of the wave is observed and listed in the below resulted statistics.

\begin{tabular}{|lll|}
\hline Column 0 & mean: 316.318830 & sd: 116.467019 \\
Column 1 & mean: 848.776372 & sd: 346.492988 \\
Column 2 & mean: 2591.745202 & sd: 361.313958 \\
Column 3 & mean: 3776.036850 & sd: 431.216165 \\
\hline$-\begin{array}{l}\text { Statistics of CASRSW computed between } 0.0 \text { and } 2.809251700680272 \\
(\mathrm{n}=276) \mid\end{array}$ & & \\
\hline
\end{tabular}

The above statistical result gives the sample standard deviation for CASRSW is 136.77 and Mean (Average) of 313.5. The below statistical result gives the sample standard deviation for DASRSW is 208.62 and Mean (Average) of 183.5.

\begin{tabular}{|c|c|c|c|}
\hline & mean: & 4295 & sd: 74 \\
\hline 21 & 1 & 1556 & 1: 48 \\
\hline & 2 & 26 & d: 15 \\
\hline $\begin{array}{l}\text { Column } 3 \\
----\end{array}$ & 3 mean: & 5016.00 & sd: 22.723 \\
\hline \multicolumn{4}{|c|}{$\begin{array}{l}\text { Statistics of DASRSW computed between } 0.0 \text { and } 4.916375 \\
(n=486)\end{array}$} \\
\hline
\end{tabular}

The fig. 5 shows the frequency distribution of crackles at the different stages of wheezing and its pitch is observed. The yellowishwhite patches in the spectrogram indicate the pitch of the sound and it's almost continuous in case of the wheezing subject. These patches are discontinuous in case of crackles.

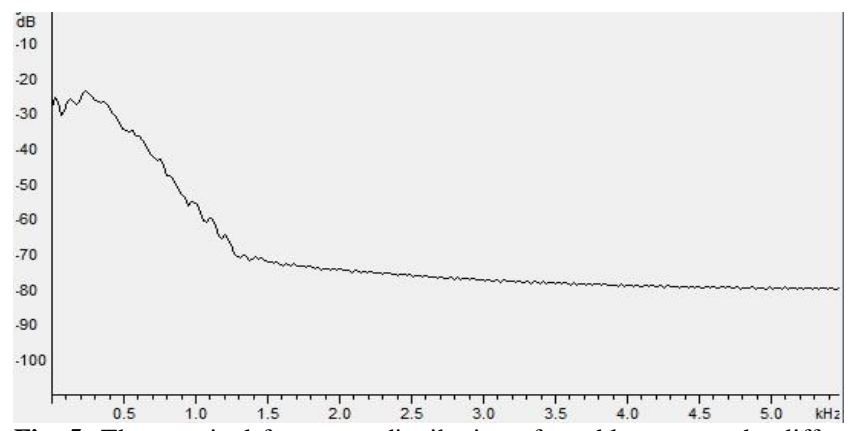

Fig. 5: The marginal frequency distribution of crackle wave at the different stages 


\section{Conclusion}

The main idea driving EEMD is very basic as it isolates sound influxes of various scales without undue mode blending [14]. EEMD utilizes all the measurable highlights of the noise encountered with wave sounds however the issue experienced with this is how to treat the multimode conveyance of the IMFs. The contrast between nonlinear multiplicative associations and emphases utilizing both EMD and EEMD were examined. The Fourier investigation for added substance commotion or Wavelets for correlative noise showed vagueness which must be recalculated to discover added substance or multiplicative [14]. The system is autonomous whether the ASRSW is straight or stationary and it is versatile in nature. The recreation of CASRSW and DASRSW are to a great degree simple. In any case, EEMD is slower contrasted with EMD is watched. Understanding of the fine data in an ASRSW of every IMF is an energizing errand. The present analysis has emphatically recommended that near and decisive parts of continuous and discontinuous unusual waves are relatively much comparable yet the varieties at the different pitch of waves with vitality and eventfulness give a better pictorial comparison. The expansion of Gaussian noise sets a global reference for EMD and EEMD technique. Finally, the headway analysis in the abnormal sound examination, EEMD turned into a standard procedure over EMD.

\section{Acknowledgement}

Thank you, RALE repository, for allowing us to use the recordings contained in its software.

\section{References}

[1] Wang, T., Zhang, M., Yu, Q., \& Zhang, H. (2012). Comparing the applications of EMD and EEMD on time-frequency analysis of seismic signal. Journal of Applied Geophysics, 83, 29-34. doi: 10.1016/j.jappgeo.2012.05.002.

[2] Sharma, B., \& Kaur, S. (2014). Distinction Between EMD \& EEMD Algorithm for Pitch Detection in Speech Processing. International Journal of Engineering Trends and Technology, 7(3), 119. 125. doi:10.14445/22315381/ijett-v7p251.

[3] Lozano, Manuel, José Antonio Fiz, and Raimon Jané. "Performance Evaluation of the Hilbert-Huang Transform for Respiratory Sound Analysis and Its Application to Continuous Adventitious Sound Characterization." Signal Processing 120 (2016): 99-116. Web.

[4] Bohadana, G., Izbicki, S.S. Kraman, Fundamentals of lung auscultation, N. Engl. J. Med. 370 (2014) 744-751.

[5] Sovijärvi A.R.A., L.P. Malmberg, G. Charbonneau, J. Vanderschoot, F. Dalmasso, C. Sacco, M. Rossi, J.E. Earis, Characteristics of breath sounds and adventitious respiratory sounds, Eur. Respir. Rev. 10 (2000) 591-59.

[6] Bouzakine, T.a., R.m. Carey, G.n. Taranhike, T.j. Eder, and R.d. Shonat. "Distinguishing between Asthma and Pneumonia through Automated Lung Sound Analysis." Proceedings of the IEEE 31st Annual Northeast Bioengineering Conference, 2005. (n.d.): n. pag. Web.

[7] Semion Kizhner, Thomas P. Flatley et al On the Huang Transform Data Processing System Development, NASA

[8] Colominas, Marcelo A., Gastón Schlotthauer, and María E. Torres. "Improved Complete Ensemble EMD: A Suitable Tool for Biomedical Signal Processing." Biomedical Signal Processing and Control 14 (2014): 19-29. Web.

[9] Sharma, R., Pachori, R. B., \& Gautam, S. (2014). Empirical Mode Decomposition Based Classification of Focal and Non-focal EEG Signals. 2014 International Conference on Medical Biometrics. doi:10.1109/icmb.2014.31.

[10] Wu, Zhaohua, and Norden E. Huang. "Ensemble Empirical Mode Decomposition: A Noise-Assisted Data Analysis Method." Advances in Adaptive Data Analysis 01.01 (2009): 1-41. Web

[11] Zhenzhen, Li, and Wu Xiaoming. "Wheeze Detection Using Fractional Hilbert Transform in the Time Domain." 2012 IEEE Biomedical Circuits and Systems Conference (BioCAS) (2012): n. pag. Web
[12] Taplidou, S. A., \& Hadjileontiadis, L. J. (2007). Wheeze detection based on time-frequency analysis of breath sounds. Computers in Biology and Medicine, 37(8), 1073-1083. doi:10.1016/j.compbiomed.2006.09.007

[13] Yamashita, M., Himeshima, M., \& Matsunaga, S. (2014). Robust classification between normal and abnormal lung sounds using adventitious-sound and heart-sound models. 2014 IEEE International Conference on Acoustics, Speech and Signal Processing (ICASSP). doi:10.1109/icassp.2014.6854437

[14] Huang, N. E., Hu, K., Yang, A. C., Chang, H., Jia, D., Liang, W.,Wu, Z. (2016). On Holo-Hilbert spectral analysis: A full informational spectral representation for nonlinear and non-stationary data. Philosophical Transactions of the Royal Society A: Mathematical, Physical and Engineering Sciences, 374(2065), 20150206. doi:10.1098/rsta.2015.0206

[15] Shankar, B. B., \& Jayadevappa, D. (2017). EMD based Hilbert energy spectrum analysis of adventitious lung wave signals. International Journal of Engineering \& Technology, 7(1-5), 122. doi:10.14419/ijet.v7i1.5.9133. 